\title{
Optical coherence tomography angiography findings in preeclampsia
}

\author{
Emine Ciloglu $\mathbb{D}^{1} \cdot$ Nefise Tanrıdan $\mathrm{Okcu}^{2} \cdot$ Neşe Çetin Dogan $\mathbb{B}^{1}$
}

Received: 20 February 2019 / Revised: 3 May 2019 / Accepted: 1 July 2019 / Published online: 17 July 2019

(c) The Author(s), under exclusive licence to The Royal College of Ophthalmologists 2019

\begin{abstract}
Objectives To investigate vascular density analysis in the superficial (SCP) and deep capillary plexus (DCP), foveal avascular zone (FAZ), peripapillary vascular density analysis (PPCVD), and peripapillary retinal nerve fiber thickness analysis (PPRNFL) using optical coherence tomography angiography (OCTA) in pregnant women with preeclampsia and to compare the obtained values with healthy pregnant and nonpregnant healthy individuals.

Methods A total of 98 pregnant women (55 had preeclampsia, 43 were healthy pregnant women) and 38 healthy nonpregnant women of reproductive age were included in the study. The patients were divided into three groups. Group 1 consisted of pregnant women with preeclampsia, group 2 comprised healthy pregnant women without preeclampsia, and group 3 consisted of healthy women who were not pregnant. All participants underwent a comprehensive ophthalmologic examination including OCTA.

Results Superficial foveal density (SFD) and deep foveal density (DFD) were lower in group 1 than in group $3(p=0.033$, $p=0.041$, respectively). Deep parafoveal density (DPD), deep temporal density (DTD), and deep superior density (DSD) values were lower in group 1 than in group 2 and group $3(p=0.001)$. PPRNFL superior and mean values were higher in group 1 and group 2 than in group 3 ( $p=0.022, p=0.029$, respectively). The mean superior and inferior RPCVD values were significantly higher in group 1 than in group 2 and group 3 ( $p=0.001, p=0.043$, and $p=0.001$, respectively).

Conclusions In preeclampsia, OCTA shows changes in the microvascular structure of the retina, even without retinopathy findings on biomicroscopy examination.
\end{abstract}

\section{Introduction}

Pregnancy causes major changes in all systems of the body. These changes affect the cardiovascular, renal, pulmonary, endocrine, metabolic, hematologic, and visual systems [1].

In a normotensive pregnant woman, the triad of systemic blood pressure of over $140 / 90 \mathrm{mmHg}$, edema, and proteinuria after week 20 of pregnancy is defined as preeclampsia. The incidence of preeclampsia is $\sim 5-7 \%$, and ocular sequelae have been reported in one in three of these patients [2].

Emine Ciloglu

drciloglu@gmail.com

1 Department of Ophthalmology, Adana City Training and Research Hospital, Adana, Turkey

2 Department of Obstetrics and Gynaecology, Adana City Training and Research Hospital, Adana, Turkey
Changes in preeclampsia-related retinopathy resemble those in hypertensive retinopathy. The most common finding is retinal arteriolar narrowing, which is usually focal but may also be generalized. Other changes include retinal hemorrhage, edema, exudate, nerve fiber layer (NFL) infarcts, and intravitreal hemorrhage secondary to neovascularization, central serous chorioretinopathy, and retinal detachment [3]. Optic nerve findings in preeclampsia are papillary edema, ischemic optic neuropathy, and optic atrophy [2]. These findings are mostly seen in the third trimester of pregnancy. Most of these findings return to normal following the resolution of preeclampsia.

Advances in imaging modalities allow for more detailed evaluation of many diseases. There are studies in the literature using optical coherence tomography (OCT) related to pregnant women. In these studies, macular thickness, choroidal thickness, and retinal nerve fiber thickness analyses were performed. Invasive procedures such as fundus fluorescein angiography (FFA) are generally avoided because the teratogenic effects are not fully known. We now 
have optical coherence tomography angiography (OCTA) devices, which can be used reliably in pregnant women. Different from FFA, this angiography method uses no intravenous contrast materials, and thus the practice is not invasive. OCTA enables high-resolution and rapid imaging of the bloodstream in various layers of the retina, it also generates a three-dimensional image of these vascular layers. Using this device, analysis can be performed of all vascular layers of the retina, foveal avascular zone (FAZ), and vascular density.

In this study, we aimed to perform vascular density analysis in the superficial (SCP) and deep capillary plexus (DCP), FAZ, peripapillary vascular density analysis (PPCVD), and peripapillary retinal nerve fiber thickness analysis (PPRNFL) using OCTA in pregnant women with preeclampsia and to compare the obtained values with healthy pregnant and nonpregnant healthy individuals.

To our knowledge the present study in which the retina was evaluated using OCTA in pregnant women is the first such report in the literature.

\section{Materials and methods}

This prospective study was performed at Adana City Training and Research Hospital. The study was performed in accordance with the tenets of the Declaration of Helsinki for research involving human subjects. The study was approved by the local ethics committee and written informed consent form was obtained from all participants.

The participants of the study included pregnant women with preeclampsia, healthy pregnant women, and nonpregnant healthy individuals. A total of 98 pregnant women and 38 healthy nonpregnant women of reproductive age were included in the study. Of the 98 pregnant women, 55 had preeclampsia and 43 were healthy pregnant women without preeclampsia. The patients were divided into three groups. Group 1 consisted of pregnant women with preeclampsia, group 2 comprised healthy pregnant women without preeclampsia, and group 3 consisted of healthy women who were not pregnant.

The common exclusion criteria for all groups were: history of diabetes and hypertension before pregnancy, history of ocular surgery, amblyopia, $>3$ diopter refractive error, presence of any retinal or optic nerve disease, glaucoma, regularly taking medication, and smoking.

The common inclusion criteria for all groups were age between 18 and 35 years, and best-corrected visual acuity $($ BCVA $)>20 / 40$. In addition, inclusion criteria were sufficiently clear ocular media, and adequate pupillary dilation and fixation to permit quality OCTA imaging.

Preeclampsia was defined as systolic blood pressure > $140 \mathrm{~mm} \mathrm{Hg}$ and/or diastolic blood pressure $>90 \mathrm{~mm} \mathrm{Hg}$ after
20 weeks of gestation in a woman with previously normal blood pressure and presence of proteinuria $(>3.0 \mathrm{~g} / 24 \mathrm{~h})$.

The preeclamptic and non-preeclamptic pregnant women were accompanied to the ophthalmology clinics by an obstetrician/gynecologist. All pregnant patients were clinically stable and capable of undergoing an OCTA examination.

All participants underwent a comprehensive ophthalmologic examination, including BCVA, slit-lamp biomicroscopy, and intraocular pressure (IOP) measurements using Goldmann applanation tonometry, central corneal thickness (CCT), dilated fundus examination, and OCTA. The measurements were performed by the same technician in the morning between the hours of 09.00-12.00 a.m. The technician did not know the clinical background of the patients.

OCTA (Optovue RTVue XR Avanti; Optovue Inc., Fremont, CA) was used for macular retinal vascularization assessments. AngioVue uses the split-spectrum amplitudedecorrelation angiography (SS-ADA) algorithm to detect erythrocyte movement. When using this software, it makes it possible to noninvasively visualize the retinal and choroidal vasculature via motion contrast. Each OCTA volume was acquired in $3 \mathrm{~s}$, and two orthogonal OCTA volumes were acquired to perform motion correction to minimize motion artifacts due to microsaccades and fixation changes. FAZ area $\left(\mathrm{mm}^{2}\right)$, superficial foveal and parafoveal VD $(\%)$, and deep foveal and parafoveal VD (\%) were evaluated. This instrument has an A-scan rate of 70,000 scans per second, uses a light source centered on $840 \mathrm{~nm}$, a bandwidth of $45 \mathrm{~nm}$, and an axial resolution of $5 \mathrm{~mm}$.

After setting the image scale to $3 \mathrm{~mm} \times 3 \mathrm{~mm}$ in the software parameters, FAZ area was measured using the 'retina' tool of the software, which delineates it automatically. The superficial retinal, deep retinal vascular networks were generated using an automated software algorithm.

The 'en face' image was then automatically segmented to define the SCP and DCP, using the built-in software segmentation algorithm. The en face images of the SCP were segmented with an inner boundary at $3 \mathrm{~mm}$ beneath the inner limiting membrane and an outer boundary at $15 \mathrm{~mm}$ beneath the inner plexiform layer (IPL). The en face images of the DCP were segmented with inner and outer boundaries at 15 and $70 \mathrm{~mm}$, respectively, beneath the IPL.

Parafoveal VD was calculated for the ring-shaped area between a 0.3 and $1.25 \mathrm{~mm}$ radius from the center of the macula. Parafoveal VD, defined as the percentage of total area occupied by vessels and microvasculature, was quantified in the SCP and DCP. The parafoveal region is divided into four sectors of $90^{\circ}$ each (temporal, superior, nasal, and inferior sectors), and VD in each sector is calculated. To calculate VD, the AngioVue Analytics software was used to extract a binary image of the blood vessels from the 
grayscale OCTA image and then the percentage of pixels with flow signal greater than the threshold in the defined region was calculated. Macular vessel densities are analyzed over a $1.5-\mathrm{mm}$ wide parafoveal, circular annulus centered on the macula.

The peripapillary images were acquired with a $4.5 \times$ 4.5-mm scanning area centered on the optic disc. The eyetracking function was activated. Motion correction to minimize motion artifacts arising from microsaccades and fixation changes was applied. The PPCVD was measured at a 1.00-mm-wide elliptical annulus extending outward from the optic disc boundary in the radial peripapillary capillary (RPC) zone. The RPC layer extends from the internal limiting membrane to the NFL.

The capillary VD percentages were automatically calculated as the proportion of the area with flowing blood vessels, defined by pixels with decorrelation values above the SS-ADA threshold level. The software version we used provides separate information on peripapillary capillary VD (only information arriving from the RPC layer capillaries is analyzed). For analyses, VD is automatically calculated for the inside-disc area, and the peripapillary area, respectively.

PPRNFL thickness was also measured using the AngioVue (Optovue, Inc.). The PPRNFL thickness was assessed at a 3.45-mm-diameter circle around the optic disc in the ONH mode.

Image quality was assessed for all OCTA scans. Poorquality images were defined as scans with quality index $<6$ or images with residual motion artifacts, segmentation errors were excluded from the analysis. Poor-quality OCTA images were characterized by doubling of vessel images and artifact lines in the target area.

\section{Statistical analysis}

Statistical analysis of the study was performed using the SPSS 20.0 (IBM Inc., Chicago, IL, USA) program. Descriptive measurements of proportional scale variables are presented as mean $\pm \mathrm{SD}$. Power analysis was performed according to the mean and standard deviation values obtained in the pilot study measurements. The smallest effect size (d1) was taken as a 5\% error margin, $90 \%$ power, and the sample size that should be in each group for three groups. The $\mathrm{G}^{*}$ Power program was used for power analysis. The consistency of continuous numeric variables to normal distribution was analyzed using the Kolmogorov-Smirnov and Shapiro-Wilk methods. Only IOP, central corneal thickness, and superficial interior density measurement results did not show normal distribution $(p<0.05)$. These variables were normalized by applying logarithmic transformation. Comparison between groups was performed using one-way analysis of variance. Tukey's honestly significant difference method was preferred as the post-hoc test for the results with significant differences. Binary comparison results are indicated in the tables using the same exponential letters. The Type-I error value was taken as 5\% and $p<0.05$ was considered statistically significant. Bonferroni correction was performed for multiple comparisons; thus, $p$ values $<0.017$ were considered statistically significant.

\section{Results}

The study included 136 eyes of 136 subjects; 55 subjects $(40.4 \%)$ in group 1,43 subjects $(31.6 \%)$ in group 2 , and 38 subjects $(28 \%)$ in group 3 . The mean age and visual acuity of all groups did not differ significantly.

In all groups, there was no pathology on the retina and optic disc related to pregnancy such as retinal hemorrhage, retina pigment epithelium changes, macular edema, serous detachment, or optic disc anomalies.

The mean gestational age of group 1 and group 2 did not differ significantly (Table 1). IOP was higher in group 1 and group 2 than in group $3(p=0.023)$. CCT was higher in group 2 than in group 3 . However, there was no statistically significant difference in terms of corrected IOP values.
Table 1 Clinical findings of all groups

\begin{tabular}{lcccc}
\hline & $\begin{array}{l}\text { Group 1 } \\
(n=55)\end{array}$ & $\begin{array}{l}\text { Group 2 } \\
(n=43)\end{array}$ & $\begin{array}{l}\text { Group 3 } \\
(n=38)\end{array}$ & $p$ \\
\hline Age (years) & $30.05 \pm 5.75$ & $31.4 \pm 5.15$ & $33.37 \pm 7.96$ & 0.182 \\
Gestational age & $35.89 \pm 2.88$ & $34.7 \pm 4.54$ & N/A & 0.223 \\
IOP & $15.42 \pm 2.91$ & $15.65 \pm 3.83$ & $13.95 \pm 1.92$ & $0.039^{*}$ \\
CCT & $526.05 \pm 27.47$ & $547.8 \pm 32.76$ & $526.42 \pm 10.05$ & $0.023^{*}$ \\
cIOP & $15.79 \pm 2.75$ & $15.46 \pm 2.86$ & $14.32 \pm 2.04$ & 0.085 \\
CMT & $206.89 \pm 15.2$ & $209.8 \pm 11.12$ & $208.68 \pm 17.3$ & 0.574 \\
\hline
\end{tabular}

The data are shown as mean $\pm \mathrm{SD}$

$I O P$ intraocular pressure, $C C T$ central corneal thickness, $C I O P$ corrected IOP, $C M T$ central macular thickness $* p<0.05$ 
Table 2 The optical coherence tomography angiography measurements of all groups

\begin{tabular}{lcccc}
\hline & $\begin{array}{l}\text { Group 1 } \\
(n=55)\end{array}$ & $\begin{array}{l}\text { Group 2 } \\
(n=43)\end{array}$ & $\begin{array}{l}\text { Group 3 } \\
(n=38)\end{array}$ & $p$ \\
\hline SFD $(\%)$ & $13.67 \pm 5.98^{\mathrm{a}}$ & $14.26 \pm 4.7$ & $16.10 \pm 5.11^{\mathrm{a}}$ & $0.033^{*}$ \\
SPD $(\%)$ & $50.9 \pm 4.06$ & $50.71 \pm 3.48$ & $51.28 \pm 3.09$ & 0.680 \\
STD $(\%)$ & $48.49 \pm 5.23$ & $48.6 \pm 4.36$ & $49.57 \pm 3.48$ & 0.601 \\
SSD $(\%)$ & $51.97 \pm 4.29$ & $52.15 \pm 4.65$ & $53.01 \pm 3.06$ & 0.877 \\
SND $(\%)$ & $50.31 \pm 4.28$ & $49.52 \pm 3.61$ & $50.45 \pm 3.35$ & 0.250 \\
SID $(\%)$ & $52.72 \pm 4.54$ & $52.6 \pm 4.32$ & $52.03 \pm 3.68$ & 0.217 \\
DFD $(\%)$ & $28.76 \pm 7.51^{\mathrm{a}}$ & $30.71 \pm 4.61$ & $32.36 \pm 6.85^{\mathrm{a}}$ & $0.041^{*}$ \\
DPD $(\%)$ & $55.56 \pm 2.8^{\mathrm{ab}}$ & $57.83 \pm 4.31^{\mathrm{a}}$ & $57.12 \pm 2.63^{\mathrm{b}}$ & $0.001^{*}$ \\
DTD $(\%)$ & $54.38 \pm 3.43^{\mathrm{ab}}$ & $57.89 \pm 3.83^{\mathrm{a}}$ & $56.58 \pm 4.56^{\mathrm{b}}$ & $0.001^{*}$ \\
DSD $(\%)$ & $54.12 \pm 5.16^{\mathrm{ab}}$ & $56.13 \pm 4.56^{\mathrm{a}}$ & $57.02 \pm 4.75^{\mathrm{b}}$ & $0.001^{*}$ \\
DND $(\%)$ & $56.2 \pm 3.15^{\mathrm{a}}$ & $57.88 \pm 4.17^{\mathrm{a}}$ & $57.31 \pm 3.53$ & $0.021^{*}$ \\
DID $(\%)$ & $56.75 \pm 3.29$ & $57.54 \pm 4.88$ & $57.7 \pm 2.53$ & 0.259 \\
FAZ $\left.(m)^{2}\right)$ & $0.35 \pm 0.06$ & $0.35 \pm 0.11$ & $0.32 \pm 0.11$ & 0.464 \\
PPRNFL mean $(\mu \mathrm{m})$ & $103.73 \pm 11.61^{\mathrm{a}}$ & $104.85 \pm 11.95^{\mathrm{b}}$ & $97.82 \pm 9.78^{\mathrm{ab}}$ & $0.029^{*}$ \\
PPRNFL superior $(\mu \mathrm{m})$ & $103.65 \pm 11.66^{\mathrm{a}}$ & $104.7 \pm 11.73^{\mathrm{b}}$ & $96.89 \pm 11.14^{\mathrm{ab}}$ & $0.022^{*}$ \\
PPRNFL inferior $(\mu \mathrm{m})$ & $103.56 \pm 12.82$ & $106.4 \pm 13.71$ & $98.68 \pm 10.01$ & 0.057 \\
RPCVD mean $(\%)$ & $54.19 \pm 2.59^{\mathrm{ab}}$ & $51.77 \pm 2.01^{\mathrm{a}}$ & $52.64 \pm 2.78^{\mathrm{b}}$ & $0.001^{*}$ \\
RPCVD superior $(\%)$ & $54.28 \pm 3.39^{\mathrm{ab}}$ & $52.2 \pm 2.5^{\mathrm{a}}$ & $52.76 \pm 3.28^{\mathrm{b}}$ & $0.043^{\mathrm{a}}$ \\
RPCVD inferior $(\%)$ & $54.22 \pm 2.37^{\mathrm{ab}}$ & $51.2 \pm 2.88^{\mathrm{a}}$ & $52.47 \pm 2.77^{\mathrm{b}}$ & $0.001^{*}$ \\
RPCID $(\%)$ & $53.04 \pm 4.78$ & $51.19 \pm 3.17$ & $52.07 \pm 5.98$ & 0.102 \\
\hline
\end{tabular}

The data are shown as mean \pm SD

$S F D$ superficial foveal density, $S P D$ superior parafoveal density, $S T D$ superficial temporal density, $S S D$ superficial superior density, $S N D$ superficial nasal density, SID superficial inferior density, $D F D$ deep foveal density, $D P D$ deep parafoveal density, $D T D$ deep temporal density, $D S D$ deep superior density, $D N D$ deep nasal density, FAZ foveal avascular zone, $P P R N F L$ peripapillary retinal nerve fiber layer, $R P C V D$ radial peripapillary capillary vessel density, $R P C I D$ radial peripapillary capillary inside disc

a, b, ab: different superscripts in a row indicate statistically significant difference $* p<0.05$
There was no statistically significant difference between the groups in terms of FAZ area $(p=0.464)$.

Superficial foveal density (SFD) and deep foveal density (DFD) were lower in group 1 than in group 3 ( $p=0.033$, $p=0.041$, respectively) (Table 2 ). Deep parafoveal density (DPD), deep temporal density (DTD), and deep superior density (DSD) values were lower in group 1 than in group 2 and $3(p=0.001)$. Deep nasal density (DND) was lower in group 1 than group $2(p=0.021)$.

PPRNFL superior and mean values were statistically significantly higher in group 1 and group 2 than in group 3 ( $p=0.022, p=0.029$, respectively). PPRNFL inferior was higher in group 1 and group 2 than in group 3 , but the difference was not statistically significant.

The mean superior and inferior RPCVD values were significantly higher in group 1 than in group 2 and group 3 ( $p=0.001, p=0.043$, and $p=0.001$, respectively).

Bonferroni correction was performed for multiple comparisons and only DPD, DTD, DSD, RPCVDI, and RPCVD variables remained statistically significant $(p<0.017)$.

\section{Discussion}

In this study, we wanted to evaluate the changes in the microvascular structure of the retina in preeclampsia by using OCTA and compared the findings with healthy pregnant and healthy nonpregnant controls.

Preeclampsia is a pregnancy-specific syndrome characterized by hypertension and proteinuria, and is associated with different symptoms such as edema, visual disturbances, headache, and epigastric pain.

Although the pathogenesis of preeclampsia is not fully elucidated, it is generally accepted that it is associated with systemic vascular resistance, and generalized vasospasm $[4,5]$. The pathophysiology of preeclampsia is similar to hypertensive retinopathy. Vasospasm, narrowing of the retinal vessels, ischemia, and microvascular permeability are known to be the underlying mechanisms.

If the vasoconstriction is severe, other changes associated with hypertensive retinopathy may occur, including retinal edema, hemorrhages, exudates, and cotton wool spots [6]. However, retinal findings may not be seen in non-severe 
vasospasm. In our study, none of the participants included in the study had any pathologic findings on biomicroscopy examination and OCTA imaging of the retina and optic disc.

In the literature, decreased IOP has been demonstrated during second and third trimesters of pregnancy. It is reported that this decrease is a result of increase in the facility of outflow via one of several possible mechanisms, including increased uveoscleral outflow due to hormonal changes and decreased venous pressure [7]. In our study, the corrected IOP values according to CCT were similar. In the preeclampsia group, the IOP values were higher than in group 3, but difference was not statistically significant. In the literature it was shown that the mean IOP values of hypertensive pregnant women in the third trimester were statistically significantly higher than those of pregnant patients who were not hypertensive [8]. In another study, it was mentioned that high systolic blood pressure caused high IOP [9].

The presence of vascular spasm and resistance in preeclampsia is expected to cause an increase in upper extremity venous pressure. Both episcleral venous pressure and IOP are increased. In this way, we can explain the high level of IOP in patients with preeclampsia. Previous studies reported that preeclampsia might affect both retinal and choroidal circulation $[10,11]$. Demir et al. found that foveal and parafoveal retinal thickness were increased, in particular in the last trimester, and this increase was thought to be due to pregnancy-related fluid retention in the retinal tissue [12]. In contrast, Atas et al. found that macular central subfield and foveal center thickness decreased in a preeclampsia group and a group with healthy pregnant compared with a healthy nonpregnant group [13]. Different from other studies, in our study there was no difference in macular thickness between the groups.

In contrast to FFA and ICG, OCTA is noncontact, noninvasive, easy to perform, and capable of providing a clear three-dimensional image of the ocular microvascular circulation. OCTA, with en face segmentation technology, provides valuable information with regard to the vessels that supply the macula and optic disc [14, 15]. OCTA employs motion contrast extracted from high-speed OCT images to produce depth-resolved, high-resolution images of retinal, and choroidal vasculature without dye injection [16].

Saiko et al. reported that OCTA clearly visualized ischemic changes in the choriocapillaris in hypertensive choroidopathy in pregnant women [17].

In this study, we wanted to evaluate whether there were microvascular changes in pregnant women using OCTA, even if there was no retinal and optic disc pathology. We found that there was a decrease in both SFD and DFD in the preeclampsia group compared with the control group. The FAZ area was wider in the pregnant group compared with the nonpregnant group, but the difference was not statistically significant.

In the DPD, DTD, and DSD, we found a significant decrease in the preeclampsia group compared with both the healthy pregnant group and the control group. There was no difference between the healthy pregnant women and the control group. According to this result, we can say that the superficial and deep plexus is not expected to be affected in healthy pregnancies with no systemic disease.

In our study, we found that there was more affect in the DCP than SCP. Multiple properties, including the distance from the larger arterioles, the proximity to the high metabolic demand of the outer retina, and the complex vascular anatomic architecture may take the DCP more susceptible to damage [18]. It is possible to say that the outer retina is more susceptible to poor perfusion caused by generalized vasospasm due to its high metabolic activity and complex vascular structure.

A few studies have evaluated RNFL thickness in preeclampsia. Neudorfer et al. reported that the average peripapillary RNFL thickness was thicker in patients with preeclampsia. They speculated that RNFL thickening in patients with preeclampsia might reflect subclinical involvement of the central nervous system [19]. Arab et al. reported that the peripapillary RNFL was reduced in women with preeclampsia and eclampsia [20].

Peripapillary RNFL thickness of the mean, superior, and inferior quadrants were higher in both the preeclampsia group and healthy pregnant group than in the control group. There was no significant difference between the preeclampsia group and the healthy pregnant group. Increased intracranial pressure in the last trimester of pregnancy may result in optic disc edema. Alternatively, it may be the result of pregnancy-induced fluid retention. Although edema was not detected in biomicroscopy examinations, the increase in thickness in RNFL can be detected at an early stage.

The RPC plexus consists of long, straight vessels with infrequent anastomoses found in the RNFL. These capillaries arise from peripapillary retinal arterioles, extend radially from the optic disc, and run in parallel with the NFL axons, furthest along the temporal arcades [21]. RPC plexus measurements have the potential of providing key functional information in disease processes such as glaucoma and other optic neuropathies [22, 23].

Quantitative studies have reported positive correlations between RPC plexus density and NFL thickness, further validating the role of the RPC plexus in maintaining NFL health [24, 25]. Thus, OCTA-derived measurements of RPC plexus could provide important information on the perfusion of the NFL, which relates to its metabolism and function in both health and disease. In our study, the RPCVD values were significantly higher in the preeclampsia group than the control group. 
No recent studies using OCTA have investigated the superficial and deep foveal and parafoveal vessel densities, FAZ, peripapillary RNFL, and RPCVD in women with preeclampsia, healthy pregnant women, and healthy nonpregnant women. In preeclampsia, OCTA shows changes in the microvascular structure of the retina, even without retinopathy findings on biomicroscopy examination. Follow-up of these patients should be performed with OCTA if possible. Further studies are needed to contribute to this issue.

\section{What was known before}

- The pathophysiology of preeclampsia is similar to hypertensive retinopathy. Retinal findings such as edema, hemorrhages can be seen. In the literature, there are several studies investigated the macular and choroidal thickness using OCT in pregnant women with or without preeclampsia.

\section{What this study adds}

- In this study, it was shown that there were microvascular changes in the retina using OCTA in preeclampsia, even if there was no retinal and optic disc pathology.

Acknowledgements We thank David Chapman for professional language editing.

\section{Compliance with ethical standards}

Conflict of interest The authors declare that they have no conflict of interest.

Publisher's note: Springer Nature remains neutral with regard to jurisdictional claims in published maps and institutional affiliations.

\section{References}

1. Carlin A, Alfirevic Z. Physiological changes of pregnancy and monitoring. Best Pr Res Clin Obstet Gynaecol. 2008;22:801-23.

2. Dinn RB, Harris A, Marcus PS. Ocular changes in pregnancy. Obstet Gynecol Surv. 2003;58:137-44.

3. Sheth BP, Mieler WF. Ocular complications of pregnancy. Curr Opin Ophthalmol. 2001;12:455-63.

4. Johannes D. The pathogenesis of pre-eclampsia: new aspects. J Perinat Med. 2000;28:464-71.

5. Redman CWG, Sargent IL. Pre-eclampsia, the placenta and the maternal systemic inflammatory response-a review. Placenta. 2003;24:21-27.

6. Hallum AV. Changes in the retinal arterioles associated with the hypertensions of pregnancy. Arch Ophthalmol. 1947;37:472-90.
7. Wang C, Li AL, Pang Y, Lei YQ, Yu L. Changes in intraocular pressure and central corneal thickness during pregnancy; a systematic review and meta-analysis. Int $\mathbf{J}$ Ophthalmol. 2017;10:1573-9.

8. Qureshi IA, Xi XR, Wu XD. Intraocular pressure trends in pregnancy and in the third trimester hypertansive patients. Acta Obstet Gynecol Scand. 1996;75:816-9.

9. Klein BE, Klein R, Knudtson MD. Intraocular pressure and systemic blood pressure: longitudinal perspective: the Beaver Dam Eye Study. Br J Ophthalmol. 2005;89:284-7.

10. Jaffe G, Schatz H. Ocular manifestations of preeclamsia. Am J Ophthalmol. 1987;103:309-15.

11. Bosco JAS. Spontaneous nontraumatic retinal detachment in pregnancy. Am J Obstet Gynecol. 1981;82:208-12.

12. Demir M, Oba E, Can E, Odabası M, Tiryaki S, Ozdal E, et al. Foveal and parafoveal retinal thickness in healthy pregnant women in their last trimester. Clin Ophthalmol. 2011;5:1397-1400.

13. Atas M, Acmaz G, Aksoy H, Demircan S, Atas F, Gulhan A, et al. Evaluation of the macula, retinal nerve fiber layer and choroid in preeclampsia, healthy pregnant and healthy non-pregnant women using spectral-domain optical coherence tomography. Hypertens Pregnancy. 2014;33:299-310.

14. Matsunaga D, Yi J, Puliafito CA, Kashani AH. OCT angiography in healthy human subjects. Ophthalmic Surg Lasers Imaging Retina. 2014;45:510-5.

15. Mansoori T, Sivaswamy J, Gamalapati JS, Agraharam SG, Balakrishna N. Measurement of radial peripapillary capillary density in the normal human retina using optical coherence tomography angiography. J Glaucoma. 2017;26:241-6.

16. De Carlo TE, Romano A, Waheed NK, Duker JS. A review of optical coherence tomography angiography (OCTA). Int J Retin Vitr. 2015;1:5.

17. Saito M, Ishibazawa A, Kinouchi R, Yoshida A. Reperfusion of the choriocapillaris observed using optical coherence tomography angiography in hypertensive choroidopathy. Int Ophthalmol. 2018;38:2205-10.

18. Nakahara T, Hoshino M, Hoshino S, Mori A, Sakamoto K, Ishii K. Structural and functional changes in retinal vasculature induced by retinal ischemia-reperfusion in rats. Exp Eye Res. 2015;135:134-45.

19. Neudorfer M, Spierer O, Goder M, Newman H, Barak S, Barak A, et al. The prevalence of retinal and optical coherence tomography findings in preeclamptic women. Retina. 2014;34:1376-83.

20. Arab M, Entezari M, Ghamary H, Ramezani A, Ashori A, Mowlazadeh A, et al. Peripapillary retinal fiber layer thickness in preeclampsia and eclamosia. Int Ophthalmol. 2018;38:2289-94.

21. Yu PK, Cringle SJ, Yu DY. Correlation between the radial peripapillary capillaries and the retinal nerve fibre layer in the normal human retina. Exp Eye Res. 2014;129:83-92.

22. Kornzweig AL, Eliasoph I, Feldstein M. Selective atrophy of the radial peripapillary capillaries in chronic glaucoma. Arch Ophthalmol. 1968;80:696-702.

23. Falavarjani KG, Sadda SR. Optical coherence tomography angiography of the optic nerve head, current situation and future perspective. J Eye Sci. 2016;1:38.

24. Yu PK, Balaratnasingam C, Xu J, Morgan WH, Mammo Z, Han $\mathrm{S}$, et al. Label-free density measurements of radial peripapillary capillaries in the human retina. PLoS ONE. 2015;10:e0135151.

25. Mase T, Ishibazawa A, Nagaoka T, Yokota H, Yoshida A. Radial peripapillary capillary network visualized using wide-field montage optical coherence tomography angiography. Invest Ophthalmol Vis Sci. 2016;57:504-10. 